\title{
China: el impacto del comercio de manufacturas en la actualización tecnológica empresarial
}

Estudio empírico sobre empresas chinas cotizables en la bolsa que comercian manufacturas

China: The Impact of Trade in Manufactured Products in Business Technology Update

Zhong Xiwei

Traducido por Lyu Xiaoxiao

\section{Resumen:}

El avance tecnológico es el núcleo del desarrollo industrial y el impuso principal de su renovación. Una de las metas principales de los países en vías de desarrollo es introducir capitales extranjeros que comercien manufacturas e incorporen tecnologías avanzadas en sus procesos, impulsando el avance técnico de las naciones receptoras. En este marco, el comercio de manufacturas ha cobrado una gran importancia en el ámbito internacional; al mismo tiempo, la renovación técnica se ha convertido en uno de los temas más estudiados.

En este artículo se presenta un análisis pormenorizado de la influencia de este comercio, desde el punto de vista de las empresas. En primer lugar, se muestra un estudio de su desarrollo y de su situación actual, tomando como objeto de investigación el caso de 40 empresas cotizables en bolsa de valores; posteriormente se expone una regresión de los datos surgida de las investigaciones empíricas, para probar su relación con la capacidad de innovación técnica. Los resultados de este trabajo indican que el tamaño de las empresas, el subsidio del gobierno y la razón de Deuda/Capital (D/C) son elementos influyentes en esta realidad. 
orientando | Temas de Asia Oriental. Sociedad, Cultura y Economía

Palabra clave: Comercio, Manufacturas, Innovación tecnológica, Desbordamiento tecnológico, Inversión en I+D.

\begin{abstract}
:
Technological advance is the core of industrial development and the main driver of its renewal. One of the main goals of the developing countries is to introduce foreign capital to expand the trade of manufactured products and incorporate advanced technologies in their processes, promoting the technical progress of the recipient countries. In this framework, trade in manufactured products has gained great importance in the international arena; at the same time, technical revolution has become one of the most studied topics.

This article presents a detailed analysis of the influence of this trade, from the point of view of companies. First, a study of its development and its current situation is shown, taking as a research object the case of 40 companies listed on the stock exchange; Subsequently, the regression of the panel data is exposed through empirical investigations, to prove the relationship between the trade of manufactured products and the capacity for technical innovation. The results of this document indicate that the size of the companies, the government subsidy and the Debt/Capital ratio $(\mathrm{D} / \mathrm{C})$ are influential elements in this reality.
\end{abstract}

Key words: Trade, Manufactures products, Technological innovation, Technological overflow, Investment in R\&D.

\title{
1. Planteamiento de la investigación
}

El desarrollo del comercio de manufacturas se ha convertido, en los últimos 40 años, en el eje del mercado en China. Juegan un papel fundamental el uso de capitales extranjeros, el perfeccionamiento de los procesos técnicos y el mejoramiento de la estructura industrial, con el objetivo de estimular el crecimiento económico y potencializar la competitividad internacional. 
Por otra parte, los países en vías de desarrollo muestran un claro interés por aprender de la tecnología avanzada ejercida por esta nación, además de su experiencia en gestión; en este sentido, dichas geografías vislumbran la difusión y la transferencia tecnológica como un motor para promover la investigación y el desarrollo (I+D).

En este contexto, resulta oportuno analizar cómo influye el comercio de este tipo de productos en la innovación técnica para el crecimiento económico de los países receptores. Sin embargo, pocas investigaciones han tratado la relación entre el comercio y la capacidad innovadora nacional en los países extranjeros.

La investigación puntual de este ámbito inició hace poco en China, por lo que los expertos en la materia no han llegado a un acuerdo. Teniendo en cuenta estos antecedentes, el presente trabajo pretende estudiar la influencia del resultado técnico atribuible al comercio de manufacturas; para conocer a profundidad su mecanismo de funcionamiento y aportar posibles soluciones a diversas problemáticas.

\section{Investigaciones previas}

En China y en todo el mundo se ha realizado una serie de investigaciones significativas sobre la relación entre el comercio de manufacturas y la modernización tecnológica. Cui Wei (2011), concluyó con su modelo teórico que cada país puede producir productos de recurso intensivo en capital si realizan entre ellos el comercio mutuo; así que este comercio facilita la modernización tecnológica.

Por un lado, ha generado la importación de bienes de capital -como equipos modernos y nuevos productos-, promoviendo la productividad. Por otro lado, dicho comercio ha profundizado el enlace entre empresas chinas e internacionales; en este sentido, el torrente tecnológico causado por las empresas ha suscitado la transformación tecnológica, la competencia, la demostración y la imitación, generando un cambio tecnológico en las empresas nacionales.

He Fugui y Zhang Yuehen (2009) utilizaron la metodología de investigación empírica para estudiar el efecto de la modernización técnica en el comercio y el desarrollo económico de la Provincia de Guangdong. Según su investigación, los efectos de la 
importación, la exportación y la inversión extranjera (IE) han sido positivos respecto al crecimiento económico. Este tipo de intercambio ha generado la modernización técnica; no obstante, subrayan que el comercio de importación tiene más efectos tecnológicos que el de exportación.

Por otra parte, Jayanthakumaran (2002) retomó el marco de análisis costo-beneficio para realizar una investigación empírica sobre las zonas de comercio de manufacturas en los países asiáticos. En este trabajo, el autor considera que los países de Asia Oriental, incluyendo China, han establecido zonas comerciales en las que los efectos de desbordamiento tecnológico local juegan un papel positivo.

En tanto, Coe y Helpman (1995), construyeron un modelo dinámico para analizar la función de la zona industrial sobre la apertura y transformación económica nacional; en su investigación indican que este comercio genera un efecto de inspiración y adaptación técnica que promueve la modernización productiva de un país.

En los últimos años se han publicado estudios que consideran que la transformación y el desarrollo del comercio exterior de China debería reducir gradualmente la proporción del comercio de manufacturas y aumentar la proporción del comercio general; además pugnan por la reducción de las industrias intensivas de mano de obra para participar en la división internacional del trabajo y del comercio y actualizar las industrias de alta tecnología, como sucede con las compañías emergentes estratégicas. (Yu y Yuan, 2016)

Otros expertos consideran que la transformación del comercio exterior no debe generar "ansiedad por lograr el éxito". Desde la perspectiva de las formas comerciales, señalan, no se debe reducir el comercio de manufacturas y aumentar el comercio general; ni abandonar el comercio de nivel "bajo", simplemente por aumentar el comercio de nivel "alto“; ni mucho menos transformar todos los sectores tradicionales en sectores de nuevas tecnologías. (Zhang, 2014)

En síntesis, el comercio de estos productos involucra varios elementos complejos. Además, este modelo de mercado influye en la innovación técnica nacional desde diferentes grados y horizontes; pero a pesar de su relevancia, sigue siendo un tema poco 
estudiando. Las investigaciones no se han enfocado en el caso particular de China, sino que parten del punto de vista de los países en vías de desarrollo; por otra parte, los estudios nacionales abordan este tópico desde una perspectiva general.

\section{Análisis de la situación actual del comercio en China}

\subsection{Estado del desarrollo}

Desde la reforma y apertura de China, el comercio exterior ha tenido un gran desarrollo. En el rápido crecimiento de este mercado, el comercio de manufacturas siempre ha desempeñado un papel fundamental. La compra-venta de estas mercancías inició su expansión en 1979; según la Aduana, el volumen de la importación y exportación en 1981 era de 2.635 mil millones de dólares. En 2007, este valor llegó hasta los 75.545.51 millones, representando el 50\% del volumen total de importación y exportación del país. Después de ese año, aunque el porcentaje bajó -manteniendo más del 30\% del volumen total-, el valor absoluto del volumen comercial se estabilizó (ver Gráfica 1). 
Gráfica 1. Suma total de importación, exportación y la tasa de contribución 2007-2015

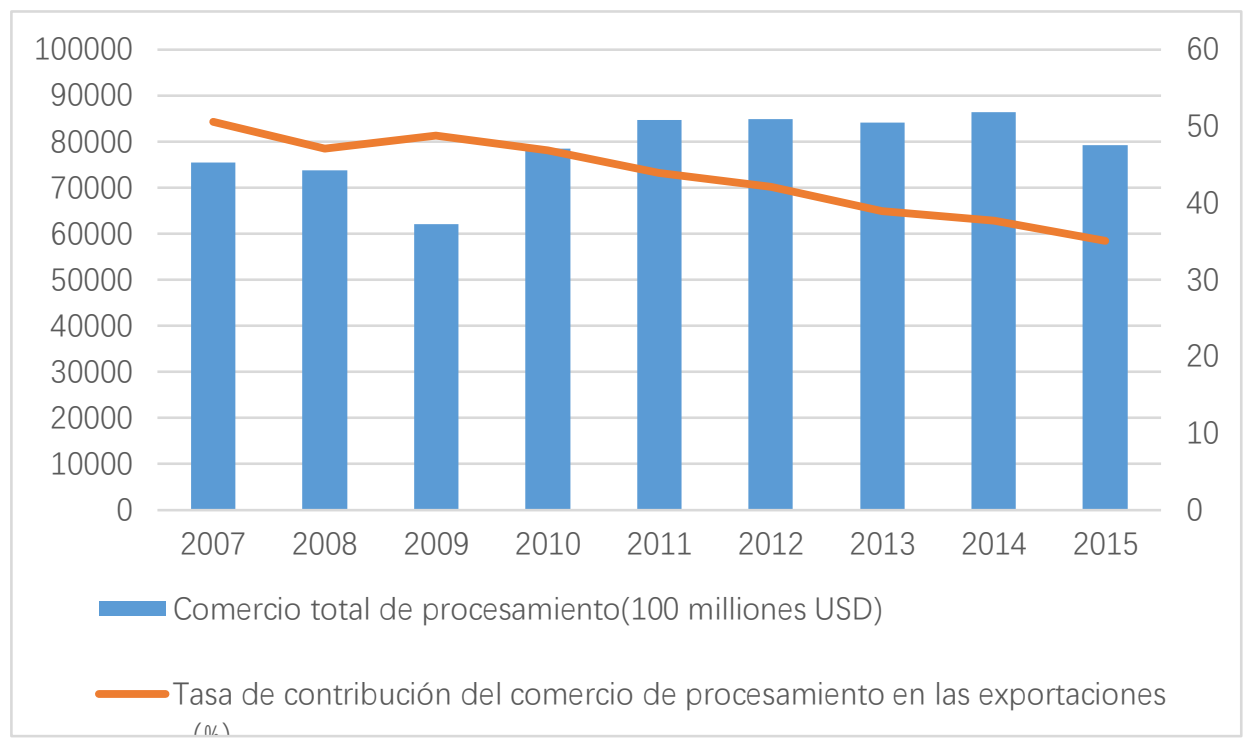

Nota: Contribución del comercio de manufacturas = exportación del comercio de productos manufacturas /exportación total del comercio exterior.

Fuente: Anuario de estadísticas de China y Anuario de estadísticas de comercio exterior de China.

\subsection{Análisis del desarrollo del comercio de manufacturas}

\section{e inversión en $I+D$ en industrias representativas}

La situación actual del desarrollo del comercio de manufacturas

de industrias representativas

Tomando en cuenta la situación actual de las empresas cotizables en la bolsa, escogimos algunas industrias representativas para este análisis: la industria textil, la de productos de plástico, la de fabricación de maquinaria, la de fabricación aeroespacial y la de equipos electrónicos. 
Tabla 1. Volumen total de industrias representativas y su porcentaje en el comercio nacional 2007-2015 (unidad: 100 millones dólares)

\begin{tabular}{|c|c|c|c|c|c|}
\hline & $\begin{array}{c}\text { Industria } \\
\text { textil }\end{array}$ & $\begin{array}{l}\text { Industria de } \\
\text { productos } \\
\text { de plástico }\end{array}$ & $\begin{array}{l}\text { Industria de } \\
\text { fabricación } \\
\text { maquinaria }\end{array}$ & $\begin{array}{l}\text { Industria de } \\
\text { fabricación } \\
\text { aeroespacial }\end{array}$ & $\begin{array}{c}\text { Industria } \\
\text { de equipos } \\
\text { electrónico } \\
\text { s }\end{array}$ \\
\hline \multirow[b]{2}{*}{2007} & 534.69 & 509.89 & 1281.74 & 5.24 & 4912.06 \\
\hline & $(5.42)$ & $(5.17)$ & $(13.0)$ & $(0.053)$ & $(49.82)$ \\
\hline \multirow{2}{*}{2008} & 542.43 & 496.29 & 1459.62 & 5.67 & 5406.36 \\
\hline & $(5.17)$ & $(4.71)$ & (13.86) & $(0.054)$ & $(51.32)$ \\
\hline \multirow{2}{*}{2009} & 458.07 & 440.91 & 1242.95 & 6.23 & 4750.43 \\
\hline & $(5.04)$ & $(4.85)$ & (13.67) & $(0.069)$ & $(52.25)$ \\
\hline \multirow[b]{2}{*}{2010} & 556.92 & 591.88 & 1565.76 & 8.34 & 6471.59 \\
\hline & $(4.81)$ & $(5.11)$ & $(13.52)$ & $(0.072)$ & $(55.90)$ \\
\hline \multirow{2}{*}{2011} & 563.98 & 688.91 & 2710.14 & 8.57 & 4934.45 \\
\hline & $(4.32)$ & $(5.28)$ & $(20.77)$ & $(0.066)$ & \\
\hline \multirow[b]{2}{*}{2012} & 542.28 & 674.28 & 2778.55 & 12.78 & 4992.6 \\
\hline & $(4.03)$ & $(5.02)$ & $(20.67))$ & $(0.095)$ & $(37.15)$ \\
\hline \multirow[b]{2}{*}{2013} & 551.69 & 682.58 & 2667.95 & 22.17 & 5141.89 \\
\hline & $(4.07)$ & $(5.03)$ & $(19.66)$ & $(0.163)$ & \\
\hline \multirow[b]{2}{*}{2014} & 525.25 & 541.78 & 2616.13 & 25.36 & 5266.3 \\
\hline & $(3.73)$ & $(3.85)$ & $(18.58)$ & $(0.180)$ & $(37.4)$ \\
\hline \multirow{2}{*}{2015} & 441.38 & 571.35 & 2295.15 & $22 / 75$ & 5344.27 \\
\hline & $(3.55)$ & $(4.59)$ & $(18.45)$ & $(0.223)$ & $(42.96)$ \\
\hline
\end{tabular}

Fuente: Anuario de estadísticas de China, Anuario de estadísticas de comercio exterior de China, Base de datos de estadísticas de desarrollo económico y social de China. 
A partir de esta tabla, podemos concluir dos características. En primer lugar, los productos mecánicos y eléctricos ocupan el sitio más alto. Los datos anteriores muestran que del año 2007 al 2015, la proporción del comercio de productos mecánicos y eléctricos abarcaba más del 50\%; en cambio, la proporción del comercio de fabricación de equipos durante del 2007 al 2010 ocupaba aproximadamente un 13\%, hasta antes del 2011, año en que registró más de 18\%; mientras que la proporción de equipos electrónicos alcanzó el 55.90\%, después de ello ha sufrido una baja moderada, pero mantiene la estabilidad.

En segundo lugar, la proporción de productos de alta densidad de mano de obra es menor y muestra una tendencia a la baja. Como puede verse en los datos, entre 2007 y 2015 la referente a la industria textil disminuyó año tras año, mientras que la proporción de productos de plástico se mantuvo en menos del $5 \%$.

Estos rasgos muestran que, en los últimos años, los productos han cambiado en el mercado; desde los tradicionales de alta densidad de mano de obra, hasta productos intensivos en capital y tecnología. Sin embargo, la magnitud de productos de alta tecnología -como los correspondientes a la industria aeroespacial- es relativamente baja.

\subsection{Análisis del estado actual de inversión en I+D}

La innovación técnica se ha convertido en un tema principal en las investigaciones sobre el comercio de productos. El gran atributo de este campo es que permite el diseño de mecanismos de motivación y aprendizaje específicos, promoviendo la confección de técnicas innovadoras y de grados de gestión avanzada. 
Tabla 2. La inversión en I+D y el porcentaje de ingresos netos 2007-2015 (unidad: 100 millones dólares, \%)

\begin{tabular}{|c|c|c|c|c|c|}
\hline & $\begin{array}{c}\text { Industria } \\
\text { textil }\end{array}$ & $\begin{array}{c}\text { Industria de } \\
\text { productos } \\
\text { de plástico }\end{array}$ & $\begin{array}{c}\text { Industria de } \\
\text { fabricación de } \\
\text { maquinaria }\end{array}$ & $\begin{array}{c}\text { Fabricación } \\
\text { aeroespacial }\end{array}$ & $\begin{array}{c}\text { Industria de } \\
\text { equipos } \\
\text { electrónicos }\end{array}$ \\
\hline 2011 & $\begin{array}{c}1360233 \\
(0.4)\end{array}$ & $\begin{array}{c}726377 \\
(0.5)\end{array}$ & $6240088(1.2)$ & 180017 & 7005706 \\
& 1380288 & 1728669 & $7041558(0.9)$ & 247090 & $(17.3)$ \\
\hline 2012 & $(0.4)$ & $(0.7)$ & & $(23)$ & $(1.9)$ \\
& 1584878 & 2279020 & $9228515(1.3)$ & 219876 & 10459083 \\
& $(0.4)$ & $(0.7)$ & & $(12.7)$ & $(2.1)$ \\
\hline 2013 & 1776979 & 416045 & $4887201 \quad(0.7)$ & 265160 & 11764875 \\
& $(0.5)$ & $(0.1)$ & & $(12.4)$ & $(2.1)$ \\
\hline 2014 & 2076652 & 2426011 & 10127297 & 269353 & 13790569 \\
& $(0.5)$ & $(0.8)$ & $(1.5)$ & $(11.8)$ & $(2.1)$ \\
\hline
\end{tabular}

Fuente: Anuario de estadísticas de ciencia y tecnología de China, Anuario de estadísticas de China.

A partir de esta información, se pueden concluir las siguientes características:

En primer lugar, la inversión en I+D de las industrias seleccionadas mostró una tendencia al alza, que indica que cada sector presta más atención a este ámbito y se esfuerza por elevar su nivel tecnológico en la producción. Al mismo tiempo, vemos que en este tipo de inversión, las industrias de textiles y plásticos ocupan un porcentaje relativamente bajo de los ingresos operativos.

En segundo lugar, la inversión en I+D en la fabricación aeroespacial representa una proporción relativamente alta de los ingresos operativos; aunque hay algunas fluctuaciones, siempre mantiene más del 10\%. Por otra parte, este tipo de financiación en la industria de equipos electrónicos es relativamente alta y ha aumentado año tras año. Por tanto, la 
industria de alta tecnología se está desarrollando rápidamente en China y recibe un fuerte apoyo gubernamental.

\section{Estudios empíricos}

\subsection{Elección de los datos variables}

\section{Medición de variables dependientes- nivel innovador tecnológico}

La medición de la innovación tecnológica es un tema central en los debates académicos. Los indicadores aplicados abarcan: la productividad total de los factores (TFP), los datos de patentes, la inversión en investigación y desarrollo (I + D), etcétera.

En este marco, Shen Kehua (2011) realizó un análisis empírico del impacto colateral de la tecnología en el comercio de manufacturas, aplicando la cantidad de solicitudes de patentes como proxy de la innovación tecnológica. Por otra parte, Zhou Xiwen (2011) estimó la productividad de factores totales de Fujian, como una medida variable de avance tecnológico local. En tanto, Su Huishuang (2007) utilizó el gasto en I+D de las empresas como una medida de innovación tecnológica.

Estos tres indicadores tienen diferentes focos de atención. Por su parte, la TFP mide el rendimiento económico de la innovación, que refleja directamente la capacidad de desarrollar y absorber nuevas tecnologías en una región; también incluye el impacto de otros factores de desbordamiento; por tanto, es una medida integral de la capacidad innovadora. En cambio, el número de patentes refleja el avance tecnológico y es una medida directa de la innovación. Por otro lado, la inversión en I+D es el principal indicador de la inversión innovadora.

Debido a que este artículo estudia el impacto del comercio de manufacturas sobre la innovación tecnológica de las empresas, desde el punto de vista microscópico, se utilizaron principalmente dos indicadores para medir la innovación tecnológica empresarial: la proporción del gasto I+D en los ingresos por ventas, como una medida del volumen de la inversión, y la proporción de I+D en el personal total, como medición de la intensidad de inversión en recursos humanos. Estos valores se analizaron tomando en cuenta la 
disponibilidad, la exactitud de los datos y la suficiencia de los indicadores de medición.

\subsection{Relación entre las variables y la intensidad del comercio}

La mayoría de las investigaciones anteriores partieron desde el punto de vista macroscópico para analizar el impacto del comercio en la innovación tecnológica de un país. Sin embargo, este trabajo se centra en una micro perspectiva.

Generalmente, los datos comerciales de procesamiento son fáciles de encontrar de acuerdo con su nivel -nacional, provincial, municipal o regional-; mientras que los datos específicos no están reflejados en el análisis de informes anuales, ni en el balance u otros documentos empresariales. Por ello, se utiliza la proporción del total de los ingresos del comercio exterior para evaluar su intensidad aproximada.

\subsection{Control de la selección de variables}

Las investigaciones nacionales e internacionales muestran que el tamaño y los subsidios gubernamentales tienen cierto impacto en la capacidad innovadora de las empresas. (Dai y Da, 2007; Fang, 2017)

En este marco, se seleccionó la escala empresarial y los subsidios gubernamentales como variables de control. Entre ellas, los activos totales de cada cierre de año y los ingresos por ventas totales forman la escala de variables, mientras que el importe de las subvenciones se relaciona con las subvenciones gubernamentales.

Por otra parte, Yang Chunming y Wu Huaqing (2011) toman a la industria de tecnología informática (TI) como ejemplo de innovación, para estudiar la relación entre la capacidad innovadora, la estructura del capital y el tamaño de la empresa. Estos autores concluyen que los tres factores se correlacionan positivamente.

En tanto, Yu Ji y Zong Haixia (2009) dedujeron que la innovación tecnológica empresarial se correlaciona negativamente con los pasivos a corto plazo y se correlaciona positivamente con los pasivos a largo plazo. Los estudios mencionados utilizan la razón Deuda/Capital (razón D/C) para medir su estructura de capital. Se puede concluir que el endeudamiento es también un factor importante de la capacidad innovadora tecnológica. 
orientando | Temas de Asia Oriental. Sociedad, Cultura y Economía

Por tanto, se seleccionaron los siguientes factores como variables de control: la escala empresarial, el subsidio gubernamental y la razón de D/C.

\section{Justificación de muestras y fuentes de datos}

\subsection{Requisitos de selección de las muestras}

Teniendo en cuenta la disponibilidad, la precisión de datos, la continuidad de tiempo e idoneidad de los indicadores de medición de las empresas cotizadas, la información retomada en este documento abarca el periodo 2011-2015. Los datos analizados abarcan las cinco industrias representantes y las 40 empresas de la muestra.

\subsection{Justificación de las fuentes de datos}

Los datos de las muestras provienen de informes anuales divulgados por el Anuario estadístico de China 2011-2015, el Anuario de ciencia y tecnología de China y los registros de las empresas, disponibles en el sitio web de Juchao. Los gastos y los datos de personal de I+D son resultado del análisis y el cálculo del Informe anual 2011-2015 de las compañías seleccionadas. Por otra parte, la información del comercio proviene principalmente de informes anuales e información pública divulgada por las muestras de 2011-2015.

Debido a que las empresas no revelaron los detalles exactos del comercio en los informes, se estimaron aproximadamente los ingresos de las principales operaciones del extranjero. Por tanto, hay una posible sobreestimación de ingresos, pero la validez objetiva es mejor que otras pruebas. Para eliminar la heterocedasticidad de las series cronológicas, se utiliza la forma logarítmica de las variables en el análisis cuantitativo. Todos los estudios empíricos en este trabajo están basados en el software Eveiws 6.0.

\subsection{Establecimiento de modelos y definición de las variables}

Los datos recopilados en este trabajo son del periodo 2011-2015. Si simplemente se usaran los datos de las series cronológicas, se enfrentaría el problema de una libertad insuficiente, y la solidez de la estimación podría verse afectada. Por tanto, aquí se analiza la influencia 
del comercio de productos procesados en la innovación tecnológica de las empresas mediante el método de los datos de panel.

Este estudio se divide en dos partes: prueba de la raíz unitaria y análisis de la regresión; según este análisis, las variables relevantes son: tamaño de la empresa, subsidios del gobierno, razón $\mathrm{D} / \mathrm{C}$, volumen del comercio, personales de $\mathrm{I}+\mathrm{D}$ y gastos de $\mathrm{I}+\mathrm{D}$; entre otras.

$$
\begin{aligned}
& R T P_{i t}=\alpha+\beta_{1} L R O I_{i t}+\beta_{2} L R A D_{i t}+\beta_{3} L E S_{i t}+\beta_{4} L G S_{i t}+\mu_{i t} \quad \text { Modelo } 1 \\
& R T E_{i t}=\alpha+\beta_{1} L R O I_{i t}+\beta_{2} L R A D_{i t}+\beta_{3} L E S_{i t}+\beta_{4} L G S_{i t}+\mu_{i t} \quad \text { Modelo } 2
\end{aligned}
$$

En estos modelos, el subíndice $i$ representa a la empresa pública, $t$ el periodo, $R$ que la variable correspondiente es el valor de la variable, $L$ el logaritmo, $R O I$ es la proporción del comercio principal en el mercado internacional del ingreso total, que indica la intensidad del comercio de manufacturas; $R A D$ es la razón de $\mathrm{D} / \mathrm{C} ; E S$ representa el tamaño empresarial, medido por los activos totales de la empresa al final del año (diez mil yuanes); $G S$ corresponde a las subvenciones del gobierno (diez mil yuanes); RTP representa la proporción de técnicos de $\mathrm{I}+\mathrm{D}$ del total empleados y $R T E$ es la proporción de los gastos de $\mathrm{I}+\mathrm{D}$ del ingreso operativo.

\subsection{Análisis de los resultados empíricos}

Prueba de raíz unitaria

Ya que la mayoría de las series cronológicas se manifiestan en sus rasgos macro, la regresión directa podría producir una conclusión falsa. Por tanto, antes de realizar el análisis es necesario realizar una prueba de raíz unitaria para juzgar la estabilidad de la secuencia; cabe aclarar que sólo se pueden incluir las series cronológicas estables.

Con el fin de garantizar la imparcialidad, la eficacia y la validez de los resultados, se emplea la prueba de Augmented Dickey-Fuller (ADF) para probar la estabilidad de las series de variables económicas. Los resultados de esta prueba son los siguientes:

Tabla 3. Resultado de la prueba de raíz unitaria ADF 
orientando | Temas de Asia Oriental. Sociedad, Cultura y Economía

\begin{tabular}{|c|c|c|c|}
\hline Variables & Estadística & Probablidad & Conclusión \\
Estabilidad & & & Sí \\
\hline LES & 178.021 & 0.0000 & Sí \\
\hline LGS & 156.452 & 0.0000 & Sí \\
\hline LRAD & 148.232 & 0.0001 & Sí \\
\hline LROI & 128.351 & 0.0007 & Sí \\
\hline LRTP & 177.652 & 0.0000 & 0.0000 \\
\hline LRTE & 165.674 & & . \\
\hline
\end{tabular}

Nota: Resultado de la prueba Fisher-ADF, obtenida desde el cálculo Eviews 6.0.

Se puede apreciar que la secuencia horizontal de cada variable ha aprobado este test, eso indica que la secuencia original es estable y que se puede realizar el análisis de regresión directamente.

\subsection{Análisis de la regresión}

Antes de realizar el análisis de la regresión, se debe determinar la forma del modelo de datos de panel. Aquí se adapta la Prueba de Hausman para determinar la elección de la forma del modelo; es decir, una selección entre el modelo de efecto fijo y el modelo de efecto aleatorio. Los resultados de la prueba son los siguientes: 
Tabla 4. Resultado de la Prueba de Hausman del Modelo 1 y Modelo 2

\begin{tabular}{|c|c|c|c|c|}
\hline & Resumen del test & Estadísticas Chi-Sq. & D.f. Chi-Sq. & Prob. \\
\hline Modelo 1 & $\begin{array}{c}\text { Sección } \\
\text { transversal } \\
\text { aleatoria }\end{array}$ & 14.367829 & 4 & 0.0068 \\
\hline Modelo 2 & $\begin{array}{c}\text { Sección } \\
\text { transversal } \\
\text { aleatoria }\end{array}$ & 12.445367 & 4 & 0.0089 \\
\hline
\end{tabular}

Nota: Resultados de la Prueba Hausman, obtenidos con el cálculo Eviews 6.0.

Se observa que las probabilidades concomitantes de la Prueba Hausman del Modelo 1 y Modelo 2 son 0.0062. y 0.0093, respectivamente, menores que 0.05; por ello se debe rechazar la hipótesis nula del modelo de efectos aleatorios. Por tanto, debe establecerse un modelo de panel de efectos fijos.

El resultado de la regresión del modelo es el siguiente:

Tabla 5. Resultado del análisis del modelo de regresión de la innovación tecnológica en las empresas que comercian manufacturas

\begin{tabular}{|c|c|c|c|}
\hline Modelo & 1 & 2 & 3 \\
\hline Número de muestras & 199 & 199 & 200 \\
\hline LES & $0.088765^{*}$ & $0.122801 * *$ & $0.129169^{* *}$ \\
& $(1.736338)$ & $(2.066341)$ & $(2.299368)$ \\
\hline LGS & $0.052489 *$ & -0.016243 & \\
& $(1.878913)$ & $(-0.868052)$ & $0.141421^{* *}$ \\
& $-0.243665^{*}$ & $0.257491 * *$ & $(2.254017)$ \\
\hline LRAD & $(-1.947329)$ & $(2.409065)$ & $0.408775^{* * *}$ \\
& $0.085675^{* *}$ & $0.404089 * * *$ & $(8.675273)$ \\
\hline LROI & $(2.022808)$ & $(7.737571)$ & 0.981767 \\
\hline
\end{tabular}




\begin{tabular}{|c|c|c|c|}
\hline F estadística & 33.6847 & 95.35135 & 202.2175 \\
\hline $\begin{array}{c}\text { Valor de P de F } \\
\text { estadística }\end{array}$ & 0.000000 & 0.000000 & 0.000000 \\
\hline Valor D. W. & 1.722857 & 2.124687 & 2.168481 \\
\hline
\end{tabular}

Nota: $* * *, * *, *$ son significativos al 1\%, 5\% y 10\%, respectivamente. Los números entre paréntesis indican valores de t-test para las variables correspondientes.

El Modelo 1 es resultado de una proporción de empleados de I+D variable y LRTP es una variable dependiente. Se puede observar que el grado de ajuste R2=0.8979 indica que el modelo es ajustable; F estadística registra un 33.6847; la probabilidad acompañante de Ftest es pequeña y las ecuaciones son significativas. Todas las variables han pasado t-test, un símbolo de coeficiente consistente con las teorías de expectativas de que las variables LES, LGS y LRAD aprueben con 10\% de significación; la variable LROI aprobó con un 5\%.

En la tabla se registra que el coeficiente LROI, correspondiente a la intensidad del comercio, es significativamente positivo; por tanto, el comercio de manufacturas tiene un efecto favorable en la intensidad del aporte del personal de I+D. El coeficiente de las variables LES, respecto al tamaño de la firma, y de las variables LGS, que reportan los subsidios del gobierno, es significativamente positivo; ello indica que, entre estas compañías, la escala se relaciona directamente con su sentido de innovación.

Por otra parte, cuando aumentan los subsidios gubernamentales, las empresas disponen de más liquidez. Una gran parte de los subsidios se dirigen a la investigación científica y a los proyectos de innovación; esto alentará enormemente a las empresas a invertir en personal de I+D. Sin embargo, los coeficientes de las variables LRAD, de activos-pasivos, es significativamente negativa, lo que indica que cuando la tasa de activos-pasivos sea más alta, la empresa reducirá la fuerza de la inversión empresarial en personal de I+D.

En gran medida, estas cifras se relacionan con la inestabilidad del desarrollo económico mundial en los últimos años. Cuando el desarrollo económico general sea estable y haya buenas perspectivas en el mercado industrial, las empresas innovadoras todavía podrán dedicarse a la inversión creativa, aún con endeudamiento, y los acreedores estarán dispuestos a financiarles, porque los acreedores valoran más la innovación 
empresarial y las perspectivas del mercado.

Por otro lado, el Modelo 2 es resultado de una regresión basada en la proporción de los costos de investigación (la proporción del ingreso total operativo en el extranjero) y toma la variable LRTE como dependiente. En la tabla se puede observar que el grado de ajuste R2= 0.962765 muestra que el modelo está bien ajustado; el estadístico $\mathrm{F}=95.35135$, es grande, lo que indica que la probabilidad complementaria de la prueba $F$ es pequeña, por ello la ecuación es significativa.

En cambio, la prueba t de la variable LGS es altamente probable y la estadística es insignificante, su correlación no es consistente con la teoría expectativa que indica que posiblemente el modelo presente una multicolinealidad. Por tanto, se toma una variable como dependiente y se considera el resto de las variables como regresión de independientes para obtener el valor de R2 y F; si el valor F aprobó la prueba, la variable y el resto de ellas tienen multicolinealidad, y viceversa. Por otra parte, se consideran las variables LGS como dependientes, las restantes como variables independientes y se realiza la regresión, consiguiendo el siguiente resultado:

$$
\mathrm{R}_{\text {LGS }}^{2}=0.889517 \quad \mathrm{~F}_{\text {LGS }}=22.22197 \quad \text { Prob }\left(\mathrm{F}_{\text {LGS }}\right)=0.000000
$$

Según la regla de juicio, existe multicolinealidad entre la variable LGS y las demás. En este modelo, las subvenciones del gobierno son variables de control que no afectan en gran medida el gasto de I+D empresarial; por ello se considera la exclusión de la variable LGS; posteriormente, con el resto de variables se realiza una regresión, registrada en la Tabla 5, en el apartado del Modelo 3.

Comparando los Modelos 2 y 3 podemos ver que los resultados de la regresión mejoran después de eliminar la variable LGS; por otro lado, el ajuste global del modelo mejora, y también incrementan las variables restantes significativamente. Si las variables aprueban la verificación $\mathrm{F}$, se puede observar desde los resultados de la regresión -excluyendo la respectiva a LGS-; estos rubros pasaron la prueba de significación y el signo del coeficiente también correspondió con el esperado. 
Por otra parte, el segmento de LROI aprobó la significación con un 1\%, registrando un coeficiente significativamente positivo; esta realidad justifica plenamente que las empresas podrían aumentar sus gastos en I+D. En cambio, LES pasó la prueba de significación del $5 \%$, este coeficiente es positivo e indica que el tamaño de las empresas tendrá un impacto positivo sobre la inversión en I+D; mientras más grande sea la empresa, hay mayor énfasis en la innovación técnica, y más costo en la inversión de I+D.

Por último, el índice de RAD pasó la prueba significativa de un 5\% y el coeficiente fue significativamente provechoso; esta tendencia también justifica que la razón $\mathrm{D} / \mathrm{C}$ (tasa de activos pasivos) de las empresas y su gasto en I + D está positivamente relacionada.

\section{Conclusiones y recomendaciones}

Los resultados de la regresión de 40 empresas cotizables en la bolsa comprueban que cuanto mayor sea el tamaño de las compañías, será mayor la fuerza corporativa de inversión en la innovación tecnológica. De igual forma, incrementará la capacidad innovadora tecnológica; que en cierta medida verifica la hipótesis de Schumpeter, que señala que las grandes empresas que disponen de más canales de financiación y poseen un fuerte control sobre el mercado, son capaces de lograr una innovación eficiente en menor tiempo. Además, sostiene que, debido al efecto del tamaño empresarial, el costo de la innovación es mucho más bajo que en las pequeñas.

Este artículo demostró que la razón $\mathrm{D} / \mathrm{C}$ de las empresas con los costos de personal de $\mathrm{I}+\mathrm{D}$ es negativa y la relación con la aportación tecnológica es positiva; esta información sugiere que su impacto es incierto. Actualmente no se ha llegado a una conclusión coherente sobre las fuentes de financiación de innovación de las empresas nacionales de comercio de manufacturas; tal vez, entre el resultado y otros impactos de la innovación tecnológica, hay cierta relevancia.

Sin embargo, las subvenciones del gobierno tienen una clara correlación positiva con la inversión en el personal de I+D. Es decir, cuando haya un aumento de los subsidios, las empresas elevarán la proporción del personal de I+D; mientras que los subsidios del gobierno no tienen una correlación con el gasto en I + D. 
Los resultados muestran que los subsidios gubernamentales hacen la operación de los fondos empresariales más flexible, y el aumento de la inversión cambia la estructura de los empleados; pero debido al retraso y a la distribución de los fondos, tal vez no pueden afectar directamente en el aumento de gastos de I+D.

\subsection{Recomendaciones políticas}

Esfuerzo estatal

En vista de que el comercio de manufacturas puede potenciar la capacidad de innovación tecnológica de las empresas, a través de diversos medios, el Estado debería tomar medidas activas para aumentar una capacidad que garantice a las empresas nacionales de comercio de productos de manufactura, a fin de aumentar los efectos de desbordamiento y de transferencia tecnológica.

En comparación con los países desarrollados, actualmente el nivel técnico y de gestión de las empresas nacionales es relativamente atrasado. El Estado debería establecer políticas y medidas correspondientes, como aumentar la apertura de las industrias relacionadas, facilitar su acceso al procesamiento, completar el sistema jurídico, enfatizar en la protección de propiedad intelectual; etcétera.

Según el desarrollo del mercado internacional, es necesario acelerar la transformación tecnológica de estas empresas chinas y aumentar su capacidad de garantía para este comercio, a fin de promover la proporción de materias primas y auxiliares, los artículos y los empaques; así como el sector intermedio, para alargar la cadena de producción industrial, aumentar el valor agregado de los productos, y fortalecer la influencia del comercio de productos en la innovación tecnológica de las empresas nacionales.

\section{Esfuerzo empresarial}

Con el mejoramiento continuo de la tecnología empresarial en los países desarrollados, el mercado internacional impone más requisitos en el nivel técnico y administrativo de las empresas de manufactura y ensamblaje. Por una parte, las empresas chinas deben participar en la certificación como CMMI y en otros sistemas calificativos para mejorar su nivel 
técnico; también deben mejorar su nivel administrativo empresarial para conseguir el reconocimiento de los clientes internacionales.

Al mismo tiempo, estas compañías deben crear y perfeccionar su mecanismo de innovación tecnológica, mejorar su capacidad de absorción tecnológica de las empresas locales y el efecto del desbordamiento tecnológico del comercio de productos manufacturados, todo ello vinculado con en el proceso de aprender, optimizar, realizar la transformación comercial, y modernizar las industrias nacionales.

Sin embargo, en la división internacional del trabajo de productos, el comercio de manufacturas de China se encuentra todavía en el extremo inferior de la cadena de valor industrial, lo cual no es propicio para la industria nacional. Por tanto, las empresas nacionales deben ser conscientes de sus deficiencias, para usar todos los recursos posibles; especialmente las ventajas de las ciudades costeras para fortalecer la cooperación con las empresas extranjeras, aplicando los nuevos conocimientos con el fin de promover el desarrollo del comercio de la tecnología electrónica informática, la biomedicina, los nuevos materiales, la protección del medio ambiente y otras industrias de alta tecnología. De esta forma, el comercio de estos productos integrará los enlaces estratégicos de alto valor añadido y de alta tecnología en la cadena de producción mundial.

\section{Bibliografía}

Coe, D. y Helpman, E. (1995) International R\&D Spillovers. European Economic Review, 39(5): 859-887.

Cui, Wei. El progreso tecnológico en el comercio de productos procesados en China: Modelo teórico y análisis empírico. Ciencias sociales de la Provincia de Henan, 2011(2):120-123.

Dai, Yueqiang y Da Qinli. (2007). Estudio empírico sobre la relación entre la estructura empresarial, la escala de capital y la inversión en innovación tecnológica. Gestión de investigación científica (5): 38-42.

Fang, Qun. (2017). El efecto del comercio de productos de procesamiento en el crecimiento económico de China [J]. Comercio International de China(5): 12-16. 
Jayanthakumaran, K. (2002). An Overview of Export Processing Zones: Selected Asian Countries. Department of Economics Working Paper Series. University of Wollongong. •

He, Fugui y Zhang, Yuehuan. (2009). Estudio sobre el efecto del comercio de productos procesados en el progreso tecnológico y el crecimiento económico de la Provincia de Guangdong. Revista de la Universidad de Huizhou (4): 38-41.

Yu, Miaojie y Yuan, Dong. (2016). Liberalización del comercio, comercio de procesamiento y costo-plus: evidencia de las empresas manufactureras de China. Mundo de gestión (9): 33-43.

Zhang, Erzhen. (2014). Transformación del comercio exterior de China: comercio de productos de procesamiento, "curva de sonrisa" y elección industrial. Investigación económica contemporánea (7): 14-18 